\title{
MODEL FOR THE TRANSIENT EMISSION FROM BLAZARS
}

\author{
AMIR LEVINSON \\ School of Physics and Astronomy, Tel Aviv University, Tel Aviv 69978, Israel
}

\section{Introduction}

The discovery of strong, variable gamma-ray sources identified with blazars by CGRO has motivated many theoretical investigations concerning the emission from relativistic jets, and several models of gamma-ray blazars have recently been developed (e.g., Dermer \& Schlickeiser 1993; Bloom \& Marscher 1993; Manneheim 1993; Sikora, et al. 1994; Blandford \& Levinson 1995). However, most of these efforts have been devoted to examine spectral properties of blazars using steady-state models, which are most suitable for exploring quiescent states. Although some progress in identifying the emission mechanisms in different types of blazars, and constraining the content and dynamics of relativistic jets on small scales has been made, it has became widely recognized that quantitative analysis of variability data is crucial for advancing our understanding of the nature of relativistic jets further. Despite recent observational efforts (e.g., Reich et al. 1993; Maraschi et al. 1994; Wagner 1996; Buckley et al. 1996; Takahashi et al. 1996 ; Mattox et al. 1997) to characterize the transient emission in blazars, only very few attempts have been made (see e.g., Romanova \& Lovelace 1997) to model variability data using time dependent models.

In the following, we briefly review some recent progress in modeling gamma-ray outbursts in blazars. In particular, a variability model in which flares are produced by radiative shocks driven by unsteady ejection of highly magnetized, relativistic fluid is considered, and some preliminary results are presented. Detailed account of the ideas discussed below will be given elsewhere (Levinson, 1997, in preparation)

\section{Radiative fronts in magnetized outflows}

Various episodes may lead to time variability of blazar emission. For example, sudden changes in particle injection rate and/or magnetic field, changes in the bulk speed, or temporal changes of the intensity of ambient radiation in ERC models. Presumably, different mechanisms would give rise to different characteristics of the time dependent emission. Comprehensive study of different variability models, and comparison of model predictions with observations is crucial for analyzing variability data. Below, we discuss a specific model of transient emission, namely dissipative fronts in relativistic, magnetized outflows. The work presented here generalizes an earlier work by Romanova \& Lovelace (1997). Although in what follows the emphasis is on the application to flares in blazars, the model is sufficiently general to be applied also to other systems involving unsteady, relativistic outflows, e.g., shock models of gamma-ray bursts (Meszaros, these proceedings).

Acceleration of a MHD outflow at a boundary (injection point) leads to excitation of waves that steepen into shocks at some distance down the injection point, and the ultimate formation of a front consisting of a pair of shocks and a contact discontinuity across which the total pressure (kinetic plus magnetic) is conserved. The position at which the front is created depends on the disturbance speed and the fluid acceleration time (or alternatively, the characteristic rate of change of the outflow parameters). The front propagates at a speed intermediate between that of the colliding streams and expands at a rate proportional to the relative velocity between the two shocks. Consequently, there is a net energy flow into the front which is balanced by either adiabatic cooling, owing to 
the front expansion, or radiative cooling, depending upon the conditions in the front. Below, we consider a situation in which the front is radiative during the initial phase of its course.

The formation, evolution and structure of such fronts have been carefully examined recently by Levinson \& van Putten (1997) in the adiabatic regime (i.e., in the absence of radiative cooling), using analytic and numerical approach. The distance $r_{o}$ from the injection point at which disturbances steepen into shocks is found to be roughly $c \Delta t \Gamma^{2} \Gamma_{A}^{2} / 3$, where $\Delta t$ is the characteristic time change of the outflow parameters (of order the dynamical time in the injection zone), $\Gamma$ is the Lorentz factor of the flow, and $\Gamma_{A}$ is the Lorentz factor associated with the Alfven speed with respect to the fluid rest frame. In the case of Poynting flux dominated outflows, rapid magnetic filed dissipation is required in order for a significant fraction of the outflow energy to be converted into kinetic energy behind the shocks. Magnetic field dissipation may be achieved in strongly turbulent MHD flows (Thompson 1997) (an ordered magnetic field component and an associated Poynting flux can still be defined), or in the case of a sudden reversal of magnetic field lines (Romanova \& Lovelace 1992). Unfortunately, the microphysics involved is far from being understood. In the model discussed below the conversion of magnetic into kinetic energy is parameterized in terms of a characteristic dissipation timescale. The results obtained by Levinson \& van Putten (1997), specifically, the formation radius $r_{o}$ and the structure of an adiabatic front, can be used to a good approximation as initial conditions for the simulation of the radiating front, provided that creation of the front occurs on a timescale much shorter than the radiative cooling time. This simple choice of initial conditions has been adopted in obtaining the results discussed below. The distribution of electrons inside the front must also be specified initially. The initial size of the front is taken to be small enough, so that the total energy loss rate experienced by the front due to cooling of the initial population of electrons is well below the rate of energy deposition in the front.

The dynamics of the system and the time evolution of the spectrum produced by the front are calculated in a self-consistent manner, by numerically solving the MHD equations governing the front dynamics coupled to the kinetic equations describing the evolution of pair, gamma-ray and synchrotron intensities. The model involves three important parameters: the dissipation rate of magnetic energy, the maximum injection energy of electrons, $E_{\text {emax }}$, and the fraction $\eta$ of dissipated energy that is tapped for the injection of electrons to $E_{\text {emax }}$. The spectrum of emitting electrons is computed by the model. At present, the only processes included are inverse Compton scattering of external radiation, and pair production on the background photons. These processes are likely to dominate the production of gamma- rays in the powerful gamma-ray AGNs. The incorporation of synchrotron and SSC processes is left for future work. In the absence of synchrotron and SSC emission, the shape of the light curve depends predominantly on the maximum injection energy of electrons, and the opacity contributed by the ambient radiation. Fluctuations in magnetic field dissipation rate may lead to substructures in the light curves.

Under the above choice of initial conditions we find, quite generally, that the front decelerates and its expansion rate decreases as the emitted energy flux rises. After the peak emission has reached the front starts accelerating and adiabatic cooling becomes gradually more important, until ultimately the initial structure and velocity of the front are restored. The rise time of the flare is related to the gradient length scale of the intensity of ambient radiation at $r_{o}$ (of order $r_{o}$ if the intensity declines as $r^{-2}$ ). The fraction of flow energy, as measured in the observer frame, that enters the front depends on the relative velocity of the colliding fluids and the front velocity. This fraction is small if the front propagates at a relativistic speed, and increases as the front decelerates. Consequently, radiative losses lead to a higher rate of energy flow into the front. This positive feedback may have important implications for the emitted spectrum, particularly in the presence of large pair production opacity, as well as for the shape of the light curve.

An example is shown in Fig. 1, where the integrated energy flux in the range $5 \mathrm{MeV}$ to 100 $\mathrm{GeV}$ is plotted as a function of the time measured by a distant observer, $t_{o b s}=\int_{0}^{t}\left[1-\beta_{s+}\left(t^{\prime}\right) \theta\right] d t^{\prime}$, where $\beta_{s+}$ is the velocity of the forward shock, and $\theta$ is the angle to the line of sight. The peak flux was reached at roughly a distance $r_{o}$ from the initial position of the front, in accordance with expectations (see discussion above). The corresponding rise time is $t_{o b s} \simeq 4 \mathrm{hrs}$. The decay time of the flare is longer by virtue of the front expansion. (The point is that the larger the front volume is, the smaller the density of injected electrons and, hence, emitted photon flux, and, therefore, the 


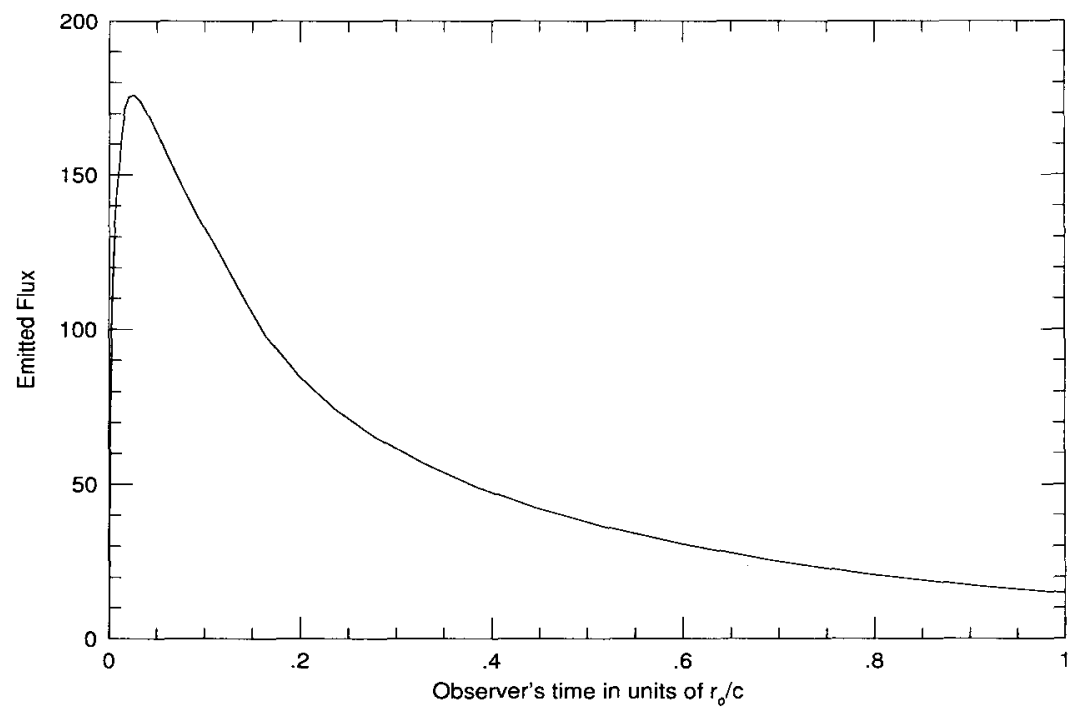

Figure 1. Typical light curve produced by a highly dissipative front in a high sigma flow $\left(\Gamma_{A}=10\right)$. Shown is the integrated energy flux in the range $5 \mathrm{MeV}$ to $100 \mathrm{GeV}$ as a function of time measured by a distant observer, for $r_{o}=10^{16} \mathrm{~cm}, \theta=0, E_{\text {emax }}=100 \mathrm{GeV}, \eta=0.9$, and Lorentz factors of the initial and injected fluids of 5 and 20 respectively. The fraction of total luminosity of external radiation that is intercepted by the front is $10^{45}$ ergs $s^{-1}$, and its spectrum was taken to be similar to those typically seen in radio quite AGN. The initial energy distribution of electrons in the front was taken to be thermal.

longer the time it takes for photons to escape the front.) This trend appears to be in agreement with that seen on several occasions (e.g., Mattox et al. 1997). Flare durations of several hours are characteristic, in this model, for a choice of parameters typical to the powerful quasars (e.g., $3 \mathrm{C} 279$ ). Application of the model to the $\mathrm{TeV}$ sources will probably require the incorporation of synchrotron and SSC processes which are likely to be more important in these objects. The emitted spectrum depends on the pair production opacity near $r_{0}$. We find, at least for the cases checked, that only a very small fraction of the radiated energy is emitted above the gamma-spheric energy at $r_{o}$. This is mainly due to the positive feedback discussed above. To examine the spectral evolution predicted by our model in the EGRET band, we divided the energy interval $10 \mathrm{MeV}$ to $10 \mathrm{GeV}$ into several subintervals. Naively one might expect the variation at high energies to be slower or later (Blandford \& Levinson 1995). However, we find roughly simultaneous flaring (with even a slight delay at lower energies). Moreover, the flux emitted at the highest energies was substantially smaller than at lower energies (as a result of attenuation by pair production of gamma-rays having energies above $\sim 0.5 \mathrm{GeV}$ during peak emission). This illustrates the cautious one must exercise in interpreting variability data using steady-state models. Further investigation is required before firm conclusions can be drown.

\section{Acknowledgment}

I thank C. Thompson, M. Van Putten and R. Blandford for useful discussions. Support by Alon fellowship is greatly acknowledged.

\section{References}

Blandford R.D. \& Levinson, A. 1995, ApJ, 441, 79

Bloom, S.D., \& Marscher, A.P. 1993, in Compton Gamma-Ray Observatory, eds. N. Gehrels \& M. Friedlander (New York: AIP), 578

Buckley, HH., et al. 1996, ApJL, 472, L9 
Dermer, C.D., \& Schlickeiser, R. 1993, ApJ, 416, 458

Ghisellini, G., \& Madau, P. 1996, MNRAS, 280, 67

Levinson, A. 1996b, ApJ, 467, 546

Levinson, A. \& Van Putten, M. 1997, ApJ, in press

Manneheim, K. 1993, A\&A, 269, 67

Maraschi, L., et al. 1994, ApJ, 435, L91

Mattox, J., et al. 1997, ApJ, 476, 692

Reich, W. et al. 1993, A\&A, 273,65

Romanova, M.M., \& Lovelace, R.V.E. 1997, ApJ, 475, 97

Romanova, M.M., \& Lovelace, R.V.E. 1992, A\&A, 262, 26

Sambruna, R.M., et al. 1997, ApJ, 474, 639

Sikora, M., Begelman, M., \& Rees, M.J. 1994, ApJ, 421, 153

Takahashi, T. et al. 1996, ApJL,470, L89

Thompson, C. 1997, in Relativistic Jets in AGN, ed. M. Ostrowski, M. Sikora, G. Madejski \& M. Begelman, in press Wagner, S.J. 1996, ApJS,120, 495 